Article

\title{
GmLecRlk, a Lectin Receptor-like Protein Kinase, Contributes to Salt Stress Tolerance by Regulating Salt-Responsive Genes in Soybean
}

\author{
Yanzheng Zhang ${ }^{\dagger}$, Qingwei Fang ${ }^{\dagger}$, Jiqiang Zheng, Zeyang Li, Yue Li, Yuan Feng, Yingpeng Han * \\ and Yongguang $\mathrm{Li} *$ (D)
}

check for

updates

Citation: Zhang, Y.; Fang, Q.; Zheng, J.; Li, Z.; Li, Y.; Feng, Y.; Han, Y.; Li, Y. GmLecRlk, a Lectin Receptor-like Protein Kinase, Contributes to Salt Stress Tolerance by Regulating Salt-Responsive Genes in Soybean. Int. J. Mol. Sci. 2022, 23, 1030 https://doi.org/10.3390/ ijms23031030

Academic Editor: Abir

U. Igamberdiev

Received: 24 December 2021

Accepted: 14 January 2022

Published: 18 January 2022

Publisher's Note: MDPI stays neutral with regard to jurisdictional claims in published maps and institutional affiliations.

Copyright: (C) 2022 by the authors. Licensee MDPI, Basel, Switzerland. This article is an open access article distributed under the terms and conditions of the Creative Commons Attribution (CC BY) license (https:// creativecommons.org/licenses/by/ $4.0 /)$.
Key Laboratory of Soybean Biology of Ministry of Education China, Key Laboratory of Soybean Biology and Breeding (Genetics) of Ministry of Agriculture and Rural Affairs, Northeast Agricultural University, Harbin 150030, China; yanzhengzhang1992@163.com (Y.Z.); fqw2536901069@163.com (Q.F.); zhengsichen1226@163.com (J.Z.); lzy780058207@163.com (Z.L.); lywcc19990804@163.com (Y.L.); z1072338704@163.com (Y.F.)

* Correspondence: hyp234286@aliyun.com (Y.H.); yongguangli@neau.edu.cn (Y.L.)

+ These authors contribute equally to this paper.

Abstract: Soybean [Glycine max (L.) Merr.] is an important oil crop that provides valuable resources for human consumption, animal feed, and biofuel. Through the transcriptome analysis in our previous study, GmLecRlk (Glyma.07G005700) was identified as a salt-responsive candidate gene in soybean. In this study, qRT-PCR analysis showed that the GmLecRlk gene expression level was significantly induced by salt stress and highly expressed in soybean roots. The $p$ CAMBIA3300-GmLecRlk construct was generated and introduced into the soybean genome by Agrobacterium rhizogenes. Compared with the wild type (WT), GmLecRlk overexpressing (GmLecRlk-ox) soybean lines had significantly enhanced fresh weight, proline (Pro) content, and catalase (CAT) activity, and reduced malondialdehyde (MDA) and $\mathrm{H}_{2} \mathrm{O}_{2}$ content under salt stress. These results show that GmLecRlk gene enhanced ROS scavenging ability in response to salt stress in soybean. Meanwhile, we demonstrated that GmLecRlk gene also conferred soybean salt tolerance when it was overexpressed alone in soybean hairy root. Furthermore, the combination of RNA-seq and qRT-PCR analysis was used to determine that GmLecRlk improves the salt tolerance of soybean by upregulating GmERF3, GmbHLH30, and GmDREB2 and downregulating GmGH3.6, GmPUB8, and GmLAMP1. Our research reveals a new mechanism of salt resistance in soybean, which exposes a novel avenue for the cultivation of saltresistant varieties.

Keywords: soybean; GmLecRlk; lectin receptor protein kinase; salt tolerance; RNA-seq

\section{Introduction}

Soybean is an important economic and oil crop, but abiotic stress, such as salinealkali stress, has a great impact on soybean yield. Although soybean is a moderately salt-tolerant crop, soybean yield can still be reduced by more than $50 \%$ in salinized land [1]. Therefore, mining salt-tolerant genes is important for improving soybean salt tolerance and cultivating new soybean varieties. All kinds of receptors located on the cell membrane or in plant cells can specifically recognize bioactive molecules and receive and process external information. The regulation of receptors is complex, including transcriptional, translational, and metabolic levels, and finally coordinates and controls plant growth and development. The translated protein in plant cells need a series of post-translational modifications, in which phosphorylation plays a key role in the process of cell life [2]. Protein phosphorylation is catalyzed by protein kinases, and a class of proteins similar to protein kinases, named receptor-like protein kinases (RLKs), are found in plants. According to the difference in the amino acid sequence of the extracellular domain of plant RLKs, 
they can be divided into six types: S-domain type [3], leucine-rich multiple sequence type (LRR) [4], epidermal growth factor-like type (EGF) [5], lectin-like type [6], tumor necrosis factor-like receptor type (TNFR) [7], and PR5-like type (PR5K) [8].

Lectin receptor protein kinases (LecRLKs) comprise a subfamily of the RLK family that consists of four domains: amino terminal signal peptide (N-terminal signal peptide), ligand binding domain (ligand-binding domain), single-channel transmembrane domain (single-pass transmembrane domain), and intracellular serine/threonine kinase domain (cytoplas-mic Ser/Thr kinase domain). LecRLKs contain a hydrophobic concave ligand binding domain that is mainly used to bind hydrophobic molecules, such as sugars, plant hormones, and cytokinins, and are important RLKs [9]. According to the diversity of extracellular lectin domains, lectin receptor protein kinases can be divided into L, G, and $C$ types [10], in which the $\mathrm{L}$ and $\mathrm{G}$ types are plant-specific, while type $\mathrm{C}$ mainly exists in mammals [11]. It has been reported that Arabidopsis thaliana, rice, and soybean contain 77, 173, and 205 LecRLKs, respectively [12].

The phylogenetic tree of the members of the GmLecRLKs family in soybean has been constructed, and the classification of the members of the family in soybean is similar to that in Arabidopsis thaliana, which was also divided into G, L, and C types. There are 138 members of type $G$ and 65 members of type $L$, while type $C$ has only two members [13]. As important receptor proteins for signal reception, LecRLKs play important roles in the response to external stress and participate in hormone signaling pathways. A small number of members also play an important role in plant growth and development. For example, overexpression of the lectin receptor protein kinase gene LecRK-V5 in Arabidopsis thaliana can inhibit the plant's response to abscisic acid (ABA), thus affecting stomatal opening and closing [14]. In leguminous plants, the PsLecRLK gene is a salt stress resistance gene that enhances the viability of plant cells by isolating the flow of sodium ions and increasing ROS to improve the salt tolerance of plants [15]. In rice, salt stress can increase the activity of SIT1 protein kinase and phosphorylate its downstream effector proteins, MAPK3 and MAPK6. In addition, the SIT1 protein can also mediate ethylene production and the ethylene signal pathway induced by salt stress [16]. The GsSRK gene, a member of the G type of the Arabidopsis LecRLK family, can be highly expressed by ABA, high salt, and drought stress, and its overexpression line survives under high salt stress; the chlorophyll content and plant height were significantly higher than those of the WT [17]. However, there are few reports on the involvement of the GmLecRlk gene in the molecular mechanism of salt stress resistance in soybean.

In a previous study, we found that overexpression of GmNFYB1 in Arabidopsis thaliana increased tolerance to abiotic stress [18], and the differentially expressed gene GmLecRlk was obtained by mining transcriptome data of WT and GmNFYB1-ox transgenic soybean, which encodes a G-type lectin receptor protein kinase. In this study, we demonstrated that the expression of the GmLecRlk gene was induced by NaCl. GmLecRlk-ox transgenic soybean was obtained by the cotyledon node transformation method, and it was found that GmLecRlk-ox transgenic soybean enhanced tolerance to salt stress and reduced the accumulation of active oxygen. It was also found that the overexpression of GmLecRlk in roots could improve the salt tolerance of soybean. It is further proved that GmLecRlk improves the salt tolerance of soybean by upregulating GmERF3, GmbHLH30, and Gm$D R E B 2$ and downregulating GmGH3.6, GmPUB8, and GmLAMP1. Our research reveals a new mechanism of salt resistance in soybean. Manipulation of these genes should facilitate improvements in salt tolerance in soybean.

\section{Results}

\subsection{Expression Pattern of GmLecRlk}

Previous studies have shown that LecRlk genes are involved in many plants' response to abiotic stress, such as Arabidopsis thaliana [14], Oryza sativa [16] and Glycine soja [17]. To explore whether the GmLecRlk gene responds to salt stress in soybean, expression patterns were identified using qRT-PCR. The results showed that the mRNA abundance of GmLecRlk 
decreased at first and then increased and reached the peak at $12 \mathrm{~h}$, which was about 40 times that of the control (Figure 1A). Further analysis of its tissue specificity showed that it was expressed in the root, stem, leaf, pod, and seed, and the highest expression level was in the roots (Figure 1B).
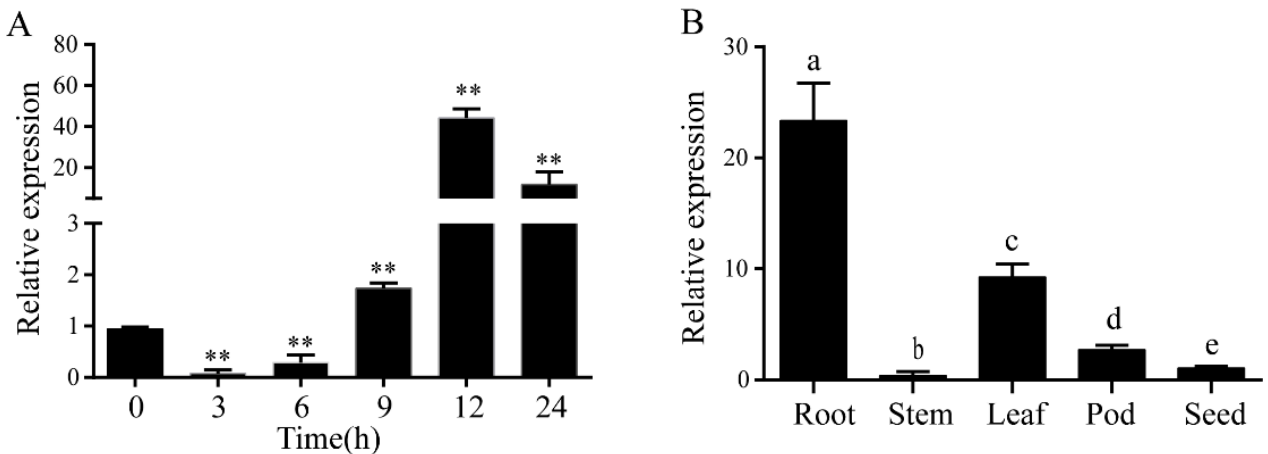

Figure 1. Expression pattern of GmLecRlk. (A) Expression analysis of GmLecRlk in response to salt stress by qRT-PCR. Asterisks indicate a significant difference compared with the corresponding control (Student's $t$ test: ${ }^{* *} p<0.01$ ). (B) Tissue specificity analysis of the GmLecRlk gene. A one-way ANOVA was used to generate the $p$ values. Error bars represent standard deviations. Values represent the means of three biological replicates.

\subsection{GmLecRlk Improves Salt Tolerance in Soybean}

Expression pattern analysis showed that the GmLecRlk gene expression level was significantly induced by salt stress. We speculated that it may be involved in the tolerance of soybean to salt stress. To verify our conjecture, overexpressing GmLecRlk transgenic soybean (GmLecRlk-ox) was used for phenotypic observation under salt stress. T-DNA region schematic diagram of the carrier (Figure 2A). GmLecRlk-ox transgenic plants were identified by DNA and RNA levels (Figure 2B,C). The selectable marker gene bar was examined using a test strip and smeared PPT (glufosinate ammonium) (Figure 2D,E). The identified homozygous T3 seeds were used in subsequent experiments.

A

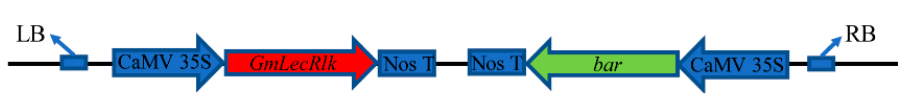

B

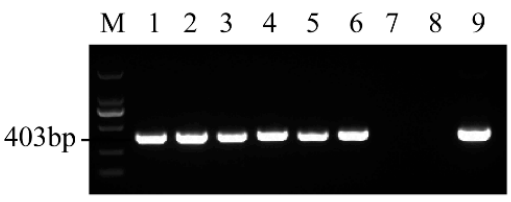

D

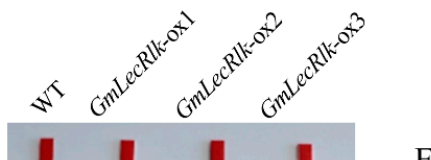

$\mathrm{C}$

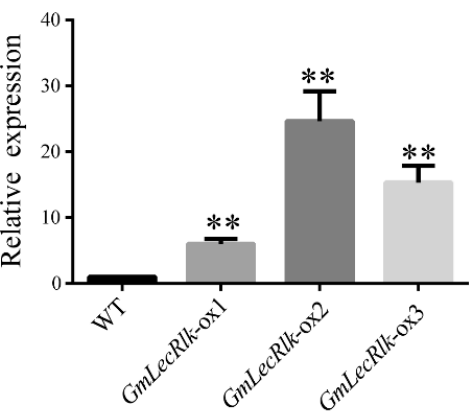

WT GmLecRlk-ox1GmLecRlk-ox2GmLecRlk-ox3

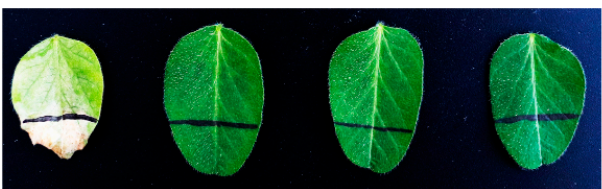

Figure 2. Identification of GmLecRlk-ox transgenic soybean. (A) Physical maps of the regions between left border (LB) and right border (RB); (B) gel image of PCR products obtained with primer sets for 
T-DNA regions of the vector. M: DL2000 marker. 1-6: positive plants 7: negative control (ddH2O). 8: DNA of WT soybean plants. 9: plasmid of the $p C A M B I A 3300-G m L e c R l k$ vector. (C) GmLecRlk gene expression level in transgenic and WT soybean by qRT-PCR. Significant differences were analyzed based on the results of three biological replications (Student's $t$ test: ${ }^{* *} p<0.01$ ). Bars indicate the standard error of the means (s.e.m). (D) Detection of the selectable marker gene bar by test strip. Blue arrow: control. Red arrow: bar is positive. (E) GmLecRlk-ox and WT soybean were screened by smearing $180 \mathrm{mg} / \mathrm{L}$ PPT on the initial leaves.

After salt treatment for 3 days, the results showed that the leaves of the WT were seriously wilted and deformed, while most of the leaves of GmLecRlk-ox had been growing vigorously (Figure 3A). The fresh weight was measured after 3 days under salt stress, and the results showed that the fresh weight of GmLecRlk-ox was significantly higher than that of the WT (Figure 3B). The ratio of variable to maximal fluorescence (Fv/Fm) is an important parameter that reflects the photosynthetic status of plants under stress. Stress damages photosystem II and reduces the efficiency of Fv/Fm [19]. The Fv/Fm value of the GmLecRlk-ox transgenic soybean was significantly higher than that of the WT soybean after 2 days of salt stress (Figure 3C). The contents of Pro and MDA were further measured, and the results showed that the Pro content of GmLecRlk-ox plants was significantly higher than that of the WT, while the content of MDA was lower than that of WT plants (Figure 3D,E).

Previous studies have shown that LecRlk is involved in a variety of signal transduction pathways. It is necessary to further study whether GmLecRlk is involved in the ROS pathway. The results of NBT and DAB staining showed that GmLecRlk-ox plants accumulated less ROS than WT (Figure 3F). The measurement of $\mathrm{H}_{2} \mathrm{O}_{2}$ content and CAT activity further confirmed our idea. The results showed that the content of $\mathrm{H}_{2} \mathrm{O}_{2}$ of GmLecRlk-ox plants was significantly lower than that of the WT (Figure 3G), while the CAT activity was higher than that of WT plants (Figure 3H). The above results show that GmLecRlk improves salt tolerance in soybean and ROS scavenging ability.

Tissue-specific expression analysis showed that the expression of GmLecRlk was the highest in roots (Figure 1B). To verify whether its expression alone in roots could also improve the resistance to salt stress, soybean hairy root composite plants overexpressing GmLecRlk were used for further analysis. We first verified the feasibility of the method, and the $p C A M B I A 3301-G U S$ transgenic hairy root composite plants were obtained. It was found that hairy roots were dyed blue by GUS staining, which proved that the method was indeed effective (Figure S1A). The GmLecRlk-ox transgenic hairy root composite plants were identified by qRT-PCR (Figure S1B,C). After salt treatment for 4 days, the results showed that the soybean leaves of empty vector (EV) began to yellow and wilt, while the GmLecRlkox hairy root compound plants had no significant change. After 7 days, some plants in the control group began to die, while the leaves of GmLecRlk-ox hairy root compound plants turned yellow, but the soybean plants still survived (Figure 4A). The fresh weight of GmLecRlk-ox was significantly higher than that of EVs under salt stress (Figure 4B). Furthermore, we measured the contents of Pro and MDA in GmLecRlk-ox and EV soybean hairy root compound plants under salt stress for 4 days. The results showed that the Pro content of GmLecRlk-ox soybean hairy root compound plant leaves was significantly higher than that of EV, while the MDA content was less increased under $200 \mathrm{mM} \mathrm{NaCl}$ (Figure 4C,D). These results confirmed our hypothesis that the overexpression of GmLecRlk in roots alone could also improve soybean tolerance to salt stress. 

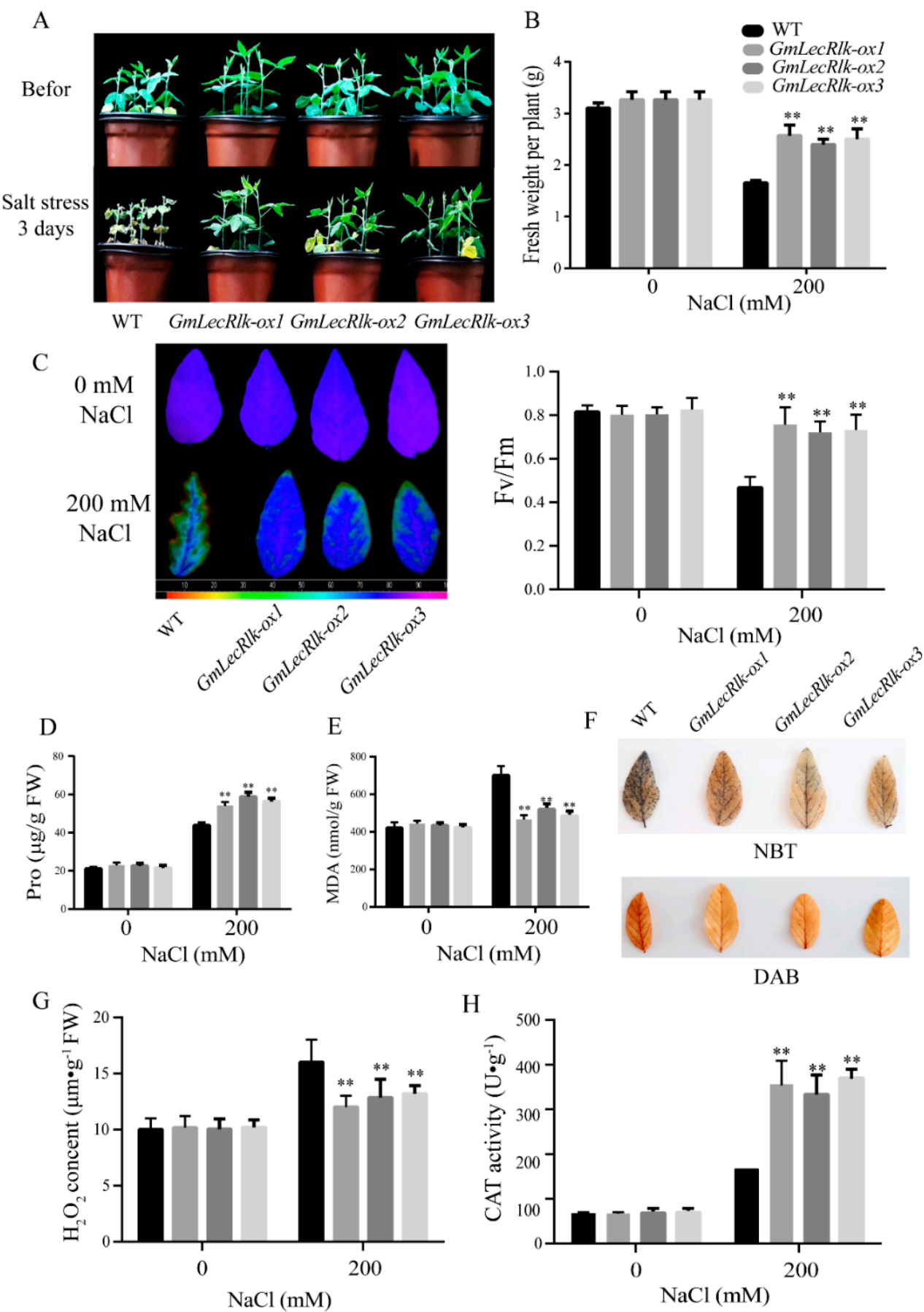

Figure 3. GmLecRlk improves salt tolerance in soybean. (A) Phenotypic analysis of GmLecRlk-ox and WT soybean under $200 \mathrm{mM} \mathrm{NaCl}$; (B) fresh weight in GmLecRlk-ox and WT soybean with 0 or $200 \mathrm{mM} \mathrm{NaCl}$; (C) Fv/Fm rate in GmLecRlk-ox and WT soybean with 0 or $200 \mathrm{mM} \mathrm{NaCl}$ (D,E,G) Pro, MDA, and $\mathrm{H}_{2} \mathrm{O}_{2}$ content in GmLecRlk-ox and WT soybean with 0 or $200 \mathrm{mM} \mathrm{NaCl}$. (F) GmLecRlk-ox and WT soybean leaves were stained with NBT and DAB under $200 \mathrm{mM} \mathrm{NaCl}$ for 3 days. (H) CAT activity in GmLecRlk-ox and WT soybean with 0 or $200 \mathrm{mM} \mathrm{NaCl}$. **, GmLecRlk-ox soybean showed a significant difference from the WT $(p<0.01)$. Error bars, s.e.m. 

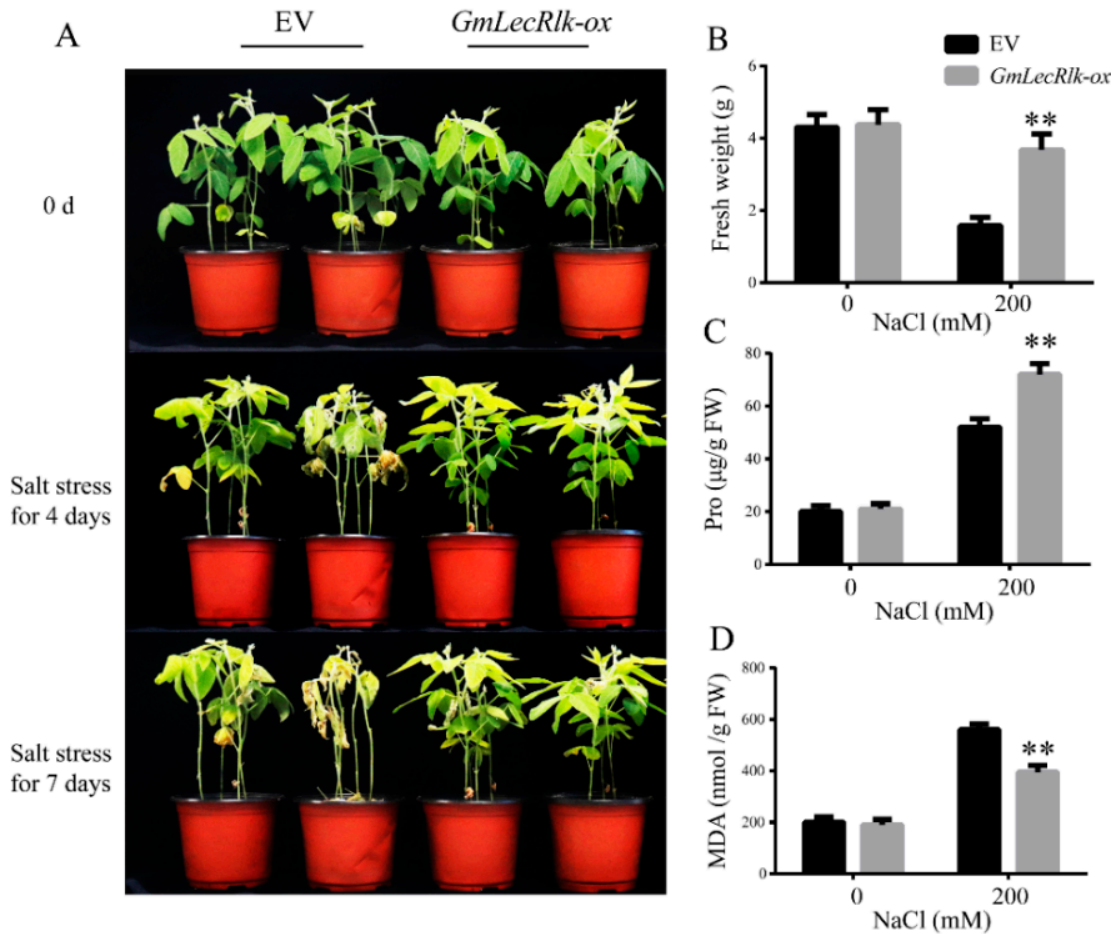

Figure 4. Phenotype identification of GmLecRlk under salt treatment in transgenic soybean hairy root compound plants. (A) Phenotypic analysis of GmLecRlk-ox and EV soybean hairy root compound plants grown under 0 or $200 \mathrm{mM} \mathrm{NaCl}$; (B) fresh weight of GmLecRlk-ox and EV soybean hairy root compound plants with 0 or $200 \mathrm{mM} \mathrm{NaCl}$ after 7 days; (C,D) pro and MDA content in GmLecRlk-ox and EV soybean hairy root compound plants with 0 or $200 \mathrm{mM} \mathrm{NaCl}$ after 4 days. ${ }^{* *}, G m L e c R l k$-ox soybean hairy root compound plants showed a significant difference from EV $(p<0.01)$. Error bars, s.e.m.

\subsection{Transcriptome Sequencing Analysis}

To identify the potential downstream genes regulated by GmLecRlk in the salt stress response, we analyzed the transcriptome of GmLecRlk-ox transgenic soybean and the WT. The results showed that 1834 DEGs were identified in GmLecRlk-ox soybean compared with the WT under non-salt stress, of which 426 genes were upregulated and 1408 genes were downregulated (Figure 5A). The results of GO enrichment of differentially expressed genes showed that biological process (BP) was mainly involved in defense response to fungus, defense response to bacterium, cellular response to hypoxia, response to jasmonic acid, and plant type secondary cell wall biogenesis. The cellular component (CC) was mainly involved in integral components of the membrane, plasma membrane, extracellular region, cell wall, and apoplast. Molecular function (MF) was mainly involved in DNA-binding transcription factor activity, heme binding, iron ion binding, oxidoreductase activity, and transmembrane transporter activity (Figure 5B). The enrichment of the KEGG metabolic pathway showed that it was mainly involved in metabolic pathways, biosynthesis of secondary metabolites, plant-pathogen interaction, phenylpropanoid biosynthesis, and flavonoid biosynthesis (Figure 5C). 

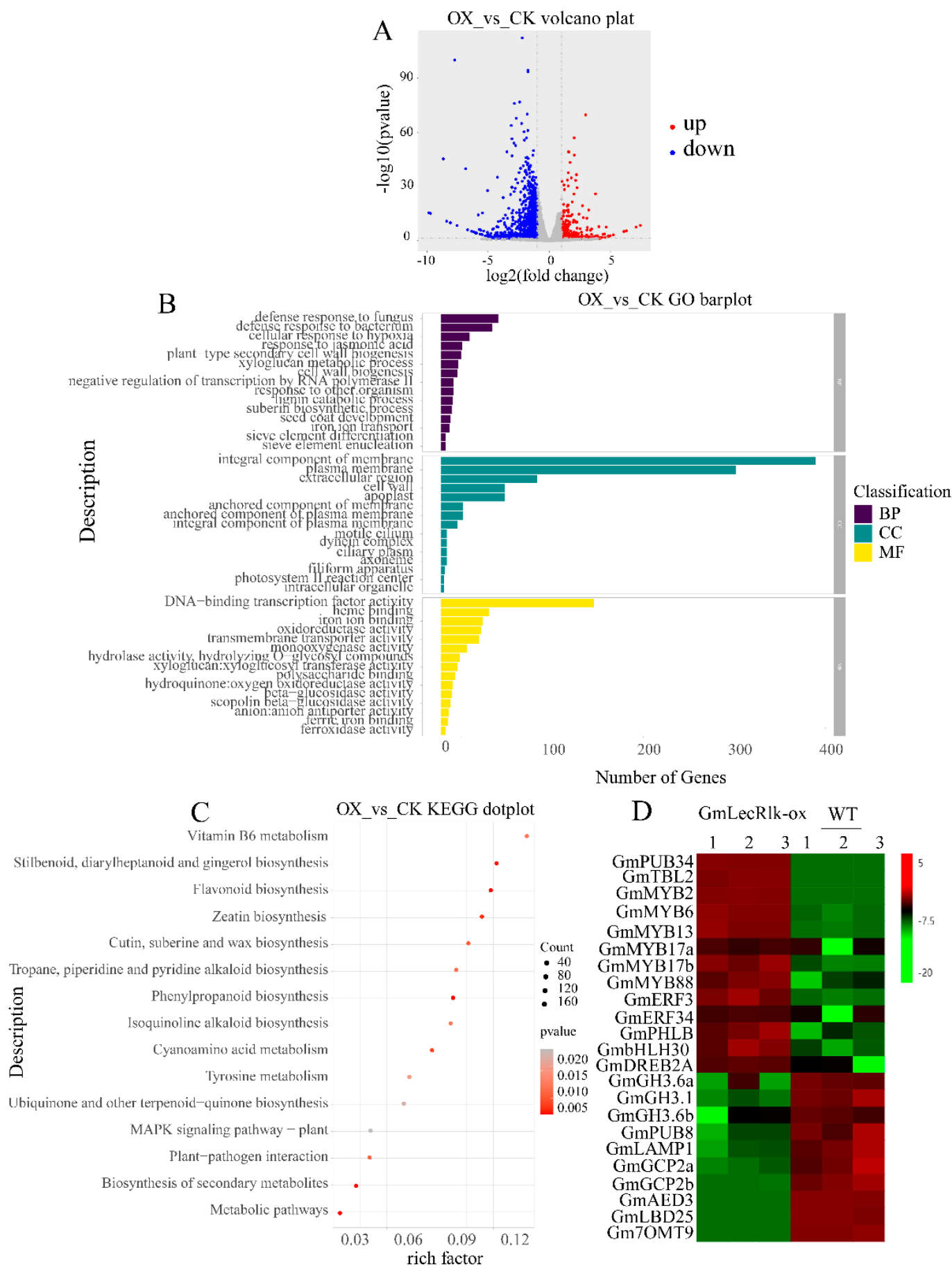

Figure 5. Transcriptomic analysis of GmLecRlk-ox and WT soybean. (A) Number of genes showing differential expression in GmLecRlk-ox and WT; (B) GO terms that were statistically enriched in differentially expressed genes in GmLecRlk-ox and WT; (C) KEGG enrichment analysis of differentially expressed genes in GmLecRlk-ox and WT plants; (D) The heat map of differential expression in GmLecRlk-ox and WT soybean. The numerical values for the green-to-red gradient bar represent the log2-fold change relative to the control sample.

Many studies have demonstrated that some TF family members, including MYB, ERF, bHLH, and DREB, are involved in the response of plants to salt stress. In addition, some negative regulatory factors are also involved in plant salt stress response, such as indole-3-acetic acid-amido synthetase (GH3.6), glutamate carboxypeptidase (AMP1), and U-box domain-containing protein (PUB). Analysis of differential genes showed that 
10 transcription factors were significantly upregulated: GmMYB2 (Glyma.09G183400), GmMYB6 (Glyma.15G144000), GmMYB13 (Glyma.09G038900), GmMYB17a (Glyma.09G261600), GmMYB17b (Glyma.10G236400), GmMYB88 (Glyma.01G044300), GmERF3 (Glyma.08G216600), GmERF34 (Glyma.01G074200), GmbHLH30 (Glyma.13G291400), and GmDREB2a (Glyma.12G103100). Seven negative regulatory factors were significantly downregulated, including three indole3-acetic acid-amido synthetase-family genes GmGH3.6a (Glyma.12G197800), GmGH3.1 (Glyma.05G101300), GmGH3.6b (Glyma.13G304000), and GmPUB8 (Glyma.15G064800) and three glutamate carboxypeptidase-family genes GmLAMP1 (Glyma.01G006700), GmGCP2a (Glyma.03G205900), and GmGCP2b (Glyma.10G088000) (Figure 5D). Differential expression of these genes suggests that GmLecRlk may improve the salt tolerance of soybean by regulating these genes.

\subsection{GmLecRlk Improves Salt Tolerance by Positively Regulating Salt Stress Response Genes}

Through the analysis of the data of differentially expressed genes in the transcriptome of GmLecRlk-ox and WT soybean, significant differences were observed in GmERF3, GmbHLH30, GmDREB2a, GmGH3.6a, GmPUB8, and GmGCP2b. To demonstrate the difference in the expression of these genes, we also measured the change in gene expression in GmLecRlk-ox and WT soybean by qRT-PCR after salt treatment for $12 \mathrm{~h}$. The results showed that the GmERF3, GmbHLH30, and GmDREB2a genes were significantly upregulated, while GmGH3.6a, GmPUB8, and GmGCP2b were significantly downregulated in GmLecRlk-ox soybean (Figure 6). Moreover, we carried out qRT-PCR verification of $G m M Y B 6, G m M Y B 13$, and $G m M Y B 17 b$, which had not been clearly reported for salt tolerance, and the most significant differences in transcriptome data were for GmPUB34 (Glyma.15G000700), GmTBL2 (Glyma.18G140800), GmAED3 (Glyma.02G213000), GmLBD25 (Glyma.19G063600), and Gm7OMT9 (Glyma.20G213600). Compared with the WT, the expression of these genes was significantly different from that of GmLecRlk-ox soybean in 0 or $200 \mathrm{mM} \mathrm{NaCl}$ (Figure S2). These results suggest that GmLecRlk participates in the response to salt stress by regulating GmERF3, GmbHLH30, GmDREB2a, GmGH3.6a, GmPUB8, GmGCP2b, or some potential regulatory factors (GmMYB6, GmMYB13, GmMYB17b, GmPUB34, GmTBL2, GmAED3, GmLBD25, and Gm7OMT9).
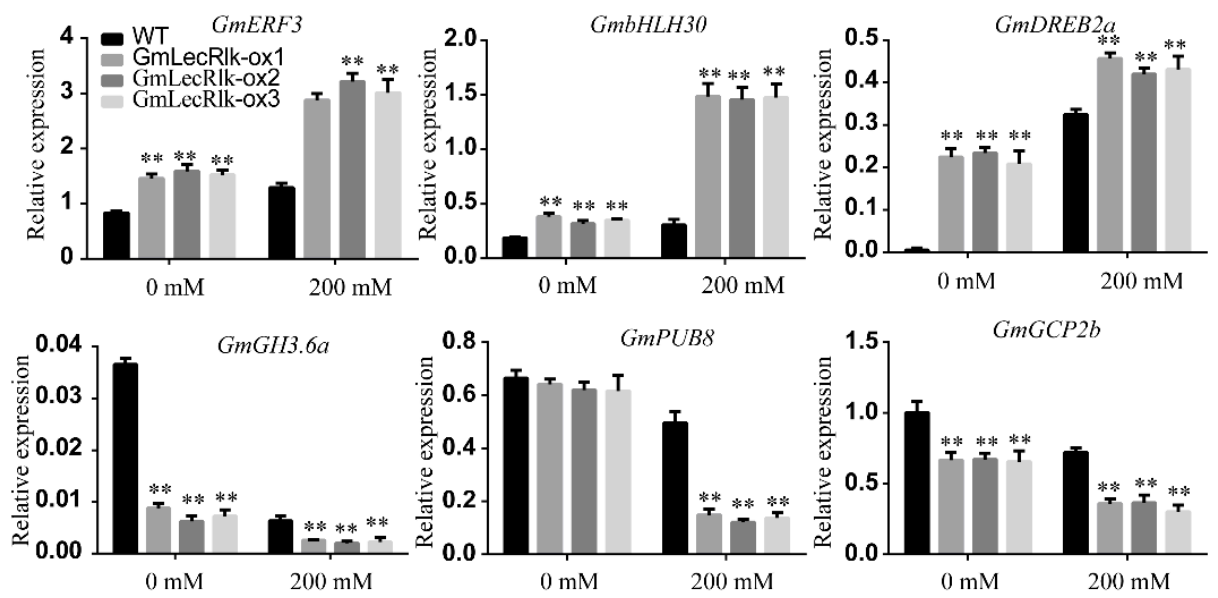

Figure 6. The transcriptional levels of GmERF3, GmbHLH30, GmDREB2a, GmGH3.6a, GmPUB8, and GmGCP2 in GmLecRlk-ox and WT soybean under 0 or $200 \mathrm{mM} \mathrm{NaCl}$ for $12 \mathrm{~h}$. ${ }^{* *}$, GmLecRlk-ox soybean showed a significant difference from the WT $(p<0.01)$. Error bars, s.e.m.

\section{Discussion}

Soybean is an important oil crop, but salt stress significantly affects soybean yield. Therefore, the identification of some salt-resistant genes and the cultivation of salt-tolerant soybean varieties can improve the resistance of soybean to salt stress and increase the use of saline-alkali land. Recent studies have shown that the S-locus LecRLK gene of Glycine soja (GsSRK) enhances salt tolerance in Arabidopsis and alfalfa by increasing SOD ac- 
tivity and scavenging ROS [20]. GmCrRLK1L20 can improve the salt tolerance of soybean by upregulating the expression of salt stress response genes, such as GmMYB84, GmWRKY40, GmDREB-like, GmGST15, GmNAC29, and GmbZIP78 [21]. Similar to the above results, GmLecRlk can also improve soybean tolerance to salt stress, but its regulatory mechanism is not very clear.

Previous studies have reported that some ERF, bHLH, and DREB transcription factors are involved in the response of plants to salt stress [21-23]. For example, GmERF3 overexpression in tobacco enhances salt resistance in plants by increasing free Pro and soluble carbohydrates [21]. The transgenic GmbHLH30 gene improves plant tolerance to aluminum by increasing the content of soluble sugar and Pro in N. benthamiana [22]. GmDREB2 enhances the salt tolerance of Arabidopsis thaliana and N. benthamiana by activating the expression of downstream salt-resistance genes [23]. Furthermore, some negative regulatory factors are also involved in the response of plants to salt stress, such as AtAMP1, which encodes a putative glutamate carboxypeptidase, and amp 1 mutants enhance salt tolerance by upregulating downstream salt-resistance genes RD29A, AHA3, and ZAT10/12 in Arabidopsis thaliana [24]. Compared with the pub23 mutant, GmPUB8 overexpression enhanced salt tolerance by upregulating eight salt-related genes in Arabidopsis thaliana [25]. AtGH3-6 encodes an indole-3-acetic acid-amidosynthetase that is induced by salt stress. Overexpressing GH3-6 in Arabidopsis thaliana negatively regulates resistance to salt stress by inhibiting the expression of RD22, KIN1, RD29A, and DREB1A [26]. In this study, transcriptome analysis showed that two ERF-family genes, a bHLH-family gene, and a DREB-family transcription factor gene were significantly upregulated, and three AMP1-family genes, three GH3-family genes, and a PUB-family gene were significantly downregulated. Based on the combination of transcriptome and qRT-PCR analyses, we determined that GmLecRlk improved the salt tolerance of soybean by upregulating the expression level of salt response genes GmERF3, GmbHLH30, and GmDREB2, and downregulating the expression level of GmGH3.6a, GmPUB8, and GmGCP2b.

In addition, some MYB family transcription factors play an important role in plant responses to salt stress. GmMYB84 enhances the salt tolerance of soybean by binding to the promoter of GmAKT1 and upregulating the expression of GmAKT1 [27]. GmMYB68 was induced by salt stress and improved the salt-alkali tolerance of soybean [28]. GsMYB15 [29], GmMYB76, GmMYB92, GmMYB177 [30], GmMYB81 [31], and GmMYB12 [32] were regulated by salt stress and improved salt tolerance in Arabidopsis thaliana. Our results showed that the expression levels of $G m M Y B 6, G m M Y B 13$, and $G m M Y B 17 b$ were induced by salt stress and were significantly upregulated in GmLecRlk-ox soybean (Figure S2). Therefore, we speculated that GmLecRlk might also enhance the salt tolerance of soybean by regulating these genes.

In this study, we found for the first time that LecRlk was involved in soybean salt stress response. It was also proved that GmLecRlk-ox enhanced the tolerance of soybean to salt stress by scavenging reactive oxygen species and positively regulating salt stress response genes. Through pot cultural experiment, it was found that the plant height and yield of GmLecRlk-ox soybean were significantly higher than those of WT under salt stress (Tables S2 and S3). WT soybeans almost never harvest under salt stress, while GmLecRlk-ox soybeans maintain a high yield. Interestingly, tissue specificity analysis showed that the expression of GmLecRLK was the highest in soybean roots, but almost undetectable in stems and seeds. Further discovery of the overexpression of GmLecRlk in soybean hairy roots alone could also improve soybean tolerance to salt stress. This finding provides a new idea for the breeding of genetically modified soybeans. As a member of the RLK family, lectin receptor protein kinases can participate in biotic and abiotic stress response and plant development regulation. However, thus far, only a small number of lectin receptor protein kinases have been reported, and their interaction factors are little known. In the future, we will use yeast library screening and mass spectrometry to identify GmLecRlk interaction factors and phosphorylation regulatory network. 


\section{Materials and Methods}

\subsection{Plant Materials and $\mathrm{NaCl}$ Stress Treatment}

Soybean stable transgenic lines were generated from the 'DongNong 50' (DN50) background. For salt stress treatment, GmLecRlk-ox and WT soybean seeds were planted in $10 \mathrm{~cm} \times 10 \mathrm{~cm}$ round plastic pots (vermiculite: peat soil is 1:3, five seeds per pot) and cultured in a greenhouse under long days (16-h light/8-h dark), $25{ }^{\circ} \mathrm{C}$, and $60 \%$ humidity. When the plant grew for 20 days, the same plants was selected to irrigate $200 \mathrm{mM} \mathrm{NaCl}$. After 3 days, the phenotype was observed, and the fresh weight of each plant was measured.

\subsection{Plasmid Construction and Plant Transformation}

To obtain GmLecRlk-overexpressing soybean, the coding sequence (CDS) of GmLecRlk was amplified by PCR from DN50 total cDNA as a template with primers GmLecRlk-F/R (Table S1) and then ligated to a large fragment of the pCAMBIA3300 vector digested by XbaI and BamHI by homologous recombination (ClonExpress II One Step Cloning Kit, Vazyme, Nanjing, China) to generate $p C A M B I A 3300-G m L e c R l k$. The recombinant plasmids were transformed into Agrobacterium rhizogenes, EHA105. According to the method described previously [33], the GmLecRlk-overexpressing DN50 transgenic soybean was obtained. Genetically modified soybeans were screened by smearing $180 \mathrm{mg} / \mathrm{L}$ glyphosate on the initial leaves and further validated by PCR (the primer bar-F/R was used for PCR identification, Table S1) and qPCR (the primer qGmLecRlk-F/R was used for qRT-PCR analysis, Table S1). Finally, a bar test strip was used to identify bar screening markers. T3 homozygous lines were used for further analysis.

To obtain transgenic soybean hairy root compound plants, $p$ CAMBIA3301-GUS, $p$ CAM$B I A 3300$ and $p C A M B I A 3300-G m L e c R l k$ were introduced into Agrobacterium rhizogenes strain K599. According to the method described previously [34], transgenic soybean hairy root compound plants were obtained and identified by qRT-PCR. When the transgenic plant complex grew to the third ternate leaf, $200 \mathrm{mM} \mathrm{NaCl}$ was used for irrigation. The phenotype was observed for 4 and 7 days, and the survival rate was counted on the 7 th day under salt stress.

\subsection{Chlorophyll Fluorescence Measurement}

The maximum efficiency of photosystem II (PSII) photochemistry (Fv/Fm ratio) was measured by modulating a chlorophyll fluorometer (Max-PAM, Heinz Walz, Effeltrich, Germany). After 20-day-old GmLecRlk-ox and WT plants were exposed to salt stress for 2 days, the leaves were collected immediately and the Fv/Fm ratio was measured. All measurements were recorded from three biological replicates.

\subsection{Measurements of Proline, Malondialdehyde, and $\mathrm{H}_{2} \mathrm{O}_{2}$ Contents}

Twenty-day-old GmLecRlk-ox and WT soybean plants were irrigated with $200 \mathrm{mM}$ $\mathrm{NaCl}$. Two days later, the leaves were collected and immediately ground on ice. Pro, malondialdehyde and $\mathrm{H}_{2} \mathrm{O}_{2}$ contents were measured by a Pro amino acid content determination kit, a malondialdehyde content determination kit, and a $\mathrm{H}_{2} \mathrm{O}_{2}$ content determination kit (Nanjing Jiancheng, Nanjing, China), respectively. The method was based on the manufacturer's protocols. All measurements were biologically repeated three times.

\subsection{Quantitative PCR Analysis}

For the analysis of the expression pattern under salt stress, the WT was placed in a black plastic bowl containing vermiculite $5 \mathrm{~cm} \times 5 \mathrm{~cm}$ (two seeds per bowl), under long-day conditions in a greenhouse (16 h light $/ 8 \mathrm{~h}$ dark), $25^{\circ} \mathrm{C}$, and $60 \%$ humidity. When the seedlings grew second ternate compound leaves, the vermiculite was washed from the roots with clean water, and they were balanced in distilled water for $24 \mathrm{~h}$. Then, the seedlings with the same growth were equally divided into two groups, one in distilled water as a control and the other in water containing $200 \mathrm{mM} \mathrm{NaCl}$ as a treatment group. Leaf samples were collected at $0,3,6,9,12$, and $24 \mathrm{~h}$ after salt treatment. 
For plant tissue specificity analysis, roots, stems, and leaves were collected from the plant at the first trifoliate stage (V1), and pods and beans were collected at the mature stage (R6). All tissues were immediately frozen in liquid nitrogen and placed in a refrigerator at $-80{ }^{\circ} \mathrm{C}$ for RNA extraction. The total RNA was extracted by RNAiso Plus (Takara, Beijing, China, 9108), and the cDNA chain was synthesized by the Prime Script RT reagent kit with gDNA Eraser (Takara, Beijing, China, RR047A), qRT-PCR was performed by using an ABI-7500 fast platform with the TB Green ${ }^{\circledR}$ Fast qPCR Mix (Takara, Beijing, China, RR430A). The program was run under the following settings: pre-denaturation at $95{ }^{\circ} \mathrm{C}$ for $30 \mathrm{~s}$, followed by a 40 -cycle program $\left(95^{\circ} \mathrm{C}, 5 \mathrm{~s} ; 60^{\circ} \mathrm{C}, 15 \mathrm{~s}\right.$; per cycle). The soybean housekeeping gene GmActin4 was used as the internal reference gene. The gene expression rate was calculated by the $2^{-\Delta \Delta C T}$ method. All experiments were analyzed with three technical and three biological replications.

\subsection{Transcriptomic Analysis}

For transcriptomic analysis, trifoliate leaves from three-week-old WT and GmLecRlk-ox transgenic soybean plants under non-salt conditions were collected and frozen in liquid nitrogen and sent to biological companies. cDNA library preparation, RNA-seq sequencing, and assembly were performed on the Illumina Nova 6000 (Illumina, San Diego, CA, USA) sequencing platform. For identification of differentially expressed genes, $\log 2$ ratios were calculated with the reads per kilobase of exon model per million mapped reads $(\mathrm{RPKM})$ value. Every gene with a $p$-value $\leq 0.05$ and a fold change $\geq 2$ was divided into upregulated and downregulated transcripts. GO annotation analyses were completed using BLAST2GO software (BioBam, Valencia, ES-Spain) (https:/ / www.blast2go.com/ [accessed on 8 October 2021]), an automated tool for the assignment of GO terms.

\subsection{Statistics of Agronomic Characters}

GmLecRlk-ox transgenic and WT soybean were planted in $20 \mathrm{~cm} \times 20 \mathrm{~cm}$ round plastic pots (peat soil, five seeds per pot). When the plant grew to the third compound leaf, it was treated with $\mathrm{NaCl}$ stress. Each pot was irrigated with $300 \mathrm{~mL} \mathrm{NaCl}$ solution with a concentration of $200 \mathrm{mM}$, and the control treatment was irrigated with the same amount of Hoagland nutrient solution. According to the development status of soybean plants, Hoagland nutrient solution was irrigated regularly to maintain its growth after salt stress treatment. The agronomic characters were counted after maturity.

\subsection{Statistical Analysis}

At least three biological replicates were included in the data, and all data were analyzed using Student's $t$ test or analysis of variance (ANOVA) for the determination of the significant differences with SPSS 25.0 (IBM, Armonk, NY, USA). All data were analyzed using GraphPad Prism 8.0.2 (GraphPad Software, San Diego, CA, USA) for calculating the means and standard error of the mean.

\section{Conclusions}

In this study, we investigated the biological functions of the soybean GmLecRlk in response to salt stress. The results show that the GmLecRlk gene enhanced ROS scavenging ability in response to salt stress in soybean. Overexpression of the GmLecRlk gene also conferred salt tolerance in soybean when it was overexpressed alone in soybean hairy root. This provides new insight into the mechanism of plant roots responding to environmental stress signals. The combination of RNA-seq and qRT-PCR analysis demonstrated that GmLecRlk improves the salt tolerance of soybean by upregulating GmERF3, GmbHLH30, and GmDREB2 and downregulating GmGH3.6, GmPUB8, and GmLAMP1. Our research reveals a new mechanism of salt stress resistance in soybean, which shows a new method for the cultivation of new salt-resistant varieties. 
Supplementary Materials: The following are available online at https://www.mdpi.com/article/10 $.3390 /$ ijms23031030/s1.

Author Contributions: Y.Z. and Q.F. performed gene cloning and RNA-seq assays. Y.Z. performed the phenotype observations and measurements. Y.Z., Q.F., J.Z., Z.L., Y.L. (Yue Li), and Y.F. performed the assay and data analysis. Y.Z., Q.F., Y.H., and Y.L. (Yongguang Li) wrote the manuscript. All authors have read and agreed to the published version of the manuscript.

Funding: This research was funded by the National Key R\&D Program of China (2021YFD1201604).

Institutional Review Board Statement: Not applicable.

Informed Consent Statement: Not applicable.

Data Availability Statement: The RNA-seq data names of the repositories and accession numbers are as follows: NCBI-SRA database under BioProject no. PRJNA788354 and accession Nos. SRR17222406, SRR17222407, SRR17222408, SRR17222409, SRR17222410, and SRR17222411.

Acknowledgments: We thank LetPub (www.letpub.com/ [accessed on 15 December 2021]) for its linguistic assistance during the preparation of this manuscript.

Conflicts of Interest: The authors declare no conflict of interest.

\section{References}

1. Lee, G.J.; Boerma, H.R.; Villagarcia, M.R.; Zhou, X.; Carter, T.E.; Li, Z.; Gibbs, M.O. A major QTL conditioning salt tolerance in S-100 soybean and descendent cultivars. Appl. Genet. 2004, 109, 1610-1619. [CrossRef] [PubMed]

2. Kersten, B.; Agrawal, G.K.; Iwahashi, H.; Rakwal, R. Plant phosphoproteomics: A long road ahead. Proteomics 2006, 6, 5517-5528. [CrossRef] [PubMed]

3. Nasrallah, J.B.; Nasrallah, M.E. Pollen[mdash]Stigma Signaling in the Sporophytic Self-Incompatibility Response. Plant Cell Online 1993, 5, 1325-1335. [CrossRef]

4. Kobe, B.; Kajava, A.V. The leucine-rich repeat as a protein recognition motif. Curr. Opin. Struct. Biol. 2001, 11, 725-732. [CrossRef]

5. Becraft, P.W.; Stinard, P.S.; McCarty, D.R. CRINKLY4: A TNFR-Like Receptor Kinase Involved in Maize Epidermal Differentiation. Science 1996, 273, 1406-1409. [CrossRef] [PubMed]

6. Hervé, C.; Dabos, P.; Galaud, J.-P.; Rougé, P.; Lescure, B. Characterization of an Arabidopsis thaliana gene that defines a new class of putative plant receptor kinases with an extracellular lectin-like domain. J. Mol. Biol. 1996, 258, 778-788. [CrossRef]

7. Wang, G.-L.; Ruan, D.-L.; Song, W.-Y.; Sideris, S.; Chen, L.; Pi, L.-Y.; Zhang, S.; Zhang, Z.; Fauquet, C.; Gaut, B.S.; et al. Xa21D encodes a receptor-like molecule with a leucine-rich repeat domain that determines race-specific recognition and is subject to adaptive evolution. Plant Cell Online 1998, 10, 765-780. [CrossRef] [PubMed]

8. Lease, K.; Ingham, E.; Walker, J.C. Challenges in understanding RLK function. Curr. Opin. Plant Biol. 1998, 1, 388-392. [CrossRef]

9. Barre, A.; Hervé, C.; Lescure, B.; Rougé, P. Lectin Receptor Kinases in Plants. Crit. Rev. Plant Sci. 2002, 21, 379-399. [CrossRef]

10. Vaid, N.; Macovei, A.; Tuteja, N. Knights in Action: Lectin Receptor-Like Kinases in Plant Development and Stress Responses. Mol. Plant 2013, 6, 1405-1418. [CrossRef]

11. Wan, J.; Patel, A.; Mathieu, M.; Kim, S.-Y.; Xu, D.; Stacey, G. A lectin receptor-like kinase is required for pollen development in Arabidopsis. Plant Mol. Biol. 2008, 67, 469-482. [CrossRef] [PubMed]

12. Vaid, N.; Pandey, P.K.; Tuteja, N. Genome-wide analysis of lectin receptor-like kinase family from Arabidopsis and rice. Plant Mol. Biol. 2012, 80, 365-388. [CrossRef] [PubMed]

13. Sun, Z.; Tian, Y.; Liu, R.; Wang, H.; Zhang, L.; Yuan, M. Genome-wide Analysis of Lectin-like Receptor Kinases(LecRLKs) Family in Soybean. Mol. Plant Breed. 2019, 17,3137-3134.

14. Desclos-Theveniau, M.; Arnaud, D.; Huang, T.-Y.; Lin, G.J.-C.; Chen, W.-Y.; Lin, Y.-C.; Zimmerli, L. The Arabidopsis Lectin Receptor Kinase LecRK-V.5 Represses Stomatal Immunity Induced by Pseudomonas syringae pv. tomato DC3000. PLOS Pathog. 2012, 8, e1002513. [CrossRef]

15. Joshi, A.; Dang, H.Q.; Vaid, N.; Tuteja, N. Pea lectin receptor-like kinase promotes high salinity stress tolerance in bacteria and expresses in response to stress in planta. Glycoconj. J. 2010, 27, 133-150. [CrossRef] [PubMed]

16. Li, C.H.; Wang, G.; Zhao, J.L.; Zhang, L.Q.; Ai, L.F.; Han, Y.F.; Sun, D.Y.; Zhang, S.W.; Sun, Y. The Receptor-Like Kinase SIT1 Mediates Salt Sensitivity by Activating MAPK3/6 and Regulating Ethylene Homeostasis in Rice. Plant Cell 2014, 26, 2538-2553. [CrossRef]

17. Sun, X.-L.; Yu, Q.-Y.; Tang, L.-L.; Ji, W.; Bai, X.; Cai, H.; Liu, X.-F.; Ding, X.-D.; Zhu, Y.-M. GsSRK, a G-type lectin S-receptor-like serine/threonine protein kinase, is a positive regulator of plant tolerance to salt stress. J. Plant Physiol. 2013, 170, 505-515. [CrossRef]

18. Li, W.; Bo, L.; Nisa, Z.; Li, Y.; Mallano, A.I.; Wang, T. Soybean GmNFYB1 transcription factor confers abiotic stress tolerance in transgenic Arabidopsis. Can. J. Plant Sci. 2016, 97, 501-515. [CrossRef] 
19. Marutani, Y.; Yamauchi, Y.; Kimura, Y.; Mizutani, M.; Sugimoto, Y. Damage to photosystem II due to heat stress without light-driven electron flow: Involvement of enhanced introduction of reducing power into thylakoid membranes. Planta 2012, 236, 753-761. [CrossRef]

20. Sun, M.; Qian, X.; Chen, C.; Cheng, S.; Jia, B.; Zhu, Y.; Sun, X. Ectopic Expression of GsSRK in Medicago sativa Reveals Its Involvement in Plant Architecture and Salt Stress Responses. Front. Plant Sci. 2018, 9, 226. [CrossRef] [PubMed]

21. Wang, Z.-Q.; Yu, T.-F.; Sun, G.-Z.; Zheng, J.-C.; Chen, J.; Zhou, Y.-B.; Chen, M.; Ma, Y.-Z.; Wei, W.-L.; Xu, Z.-S. Genome-Wide Analysis of the Catharanthus roseus RLK1-Like in Soybean and GmCrRLK1L20 Responds to Drought and Salt Stresses. Front. Plant Sci. 2021, 12, 614909. [CrossRef]

22. Song, Q.; Qian, S.F.; Chen, X.Q.; Chen, L.M.; Li, K.Z. Study on the Function of Transcription Factor GmbHLH30 on Aluminum Tolerance Preliminary in Tampa Black Soybean. Life Sci. Res. 2014, 18, 332-337.

23. Zhang, G.; Chen, M.; Li, L.; Xu, Z.; Chen, X.; Guo, J.; Ma, Y. Overexpression of the soybean GmERF3 gene, an AP2/ERF type transcription factor for increased tolerances to salt, drought, and diseases in transgenic tobacco. J. Exp. Bot. 2009, 60, 3781-3796. [CrossRef]

24. Xia, J.; He, J. Arabidopsis AMP1 Negatively Modulates Plant Responses to Salt Stress. Biotechnol. Bull. $2015,31,76-82$.

25. Wang, N.; Liu, Y.; Cong, Y.; Wang, T.; Zhong, X.; Yang, S.; Li, Y.; Gai, J. Genome-Wide Identification of Soybean U-Box E3 Ubiquitin Ligases and Roles of GmPUB8 in Negative Regulation of Drought Stress Response in Arabidopsis. Plant Cell Physiol. 2016, 57, 1189-1209. [CrossRef] [PubMed]

26. Liu, X.; Wang, R.; Jiao, B.; Dai, P.; Li, Y. Indole Acetic Acid-Amido Synthetase GH3-6 Negatively Regulates Response to Drought and Salt in Arabidopsis. Chin. Bull. Bot. 2016, 51, 586-593.

27. Zhang, W.; Wang, N.; Yang, J.; Guo, H.; Liu, Z.; Zheng, X.; Li, S.; Xiang, F. The salt-induced transcription factor GmMYB84 confers salinity tolerance in soybean. Plant Sci. 2019, 291, 110326. [CrossRef] [PubMed]

28. He, Y.; Dong, Y.; Yang, X.; Guo, D.; Qian, X.; Yan, F.; Wang, Y.; Li, J.; Wang, Q. Functional activation of a novel R2R3-MYB protein gene, GmMYB68, confers salt-alkali resistance in soybean (Glycine max L.). Genome 2020, 63, 13-26. [CrossRef] [PubMed]

29. Shen, X.-J.; Wang, Y.-Y.; Zhang, Y.-X.; Guo, W.; Jiao, Y.-Q.; Zhou, X.-A. Overexpression of the Wild Soybean R2R3-MYB Transcription Factor GsMYB15 Enhances Resistance to Salt Stress and Helicoverpa Armigera in Transgenic Arabidopsis. Int. J. Mol. Sci. 2018, 19, 3958. [CrossRef]

30. Liao, Y.; Zou, H.-F.; Wang, H.-W.; Zhang, W.-K.; Ma, B.; Zhang, J.-S.; Chen, S.-Y. Soybean GmMYB76, GmMYB92, and GmMYB177 genes confer stress tolerance in transgenic Arabidopsis plants. Cell Res. 2008, 18, 1047. [CrossRef]

31. Bian, S.; Jin, D.; Sun, G.; Shan, B.; Zhou, H.; Wang, J.; Zhai, L.; Li, X. Characterization of the soybean R2R3-MYB transcription factor GmMYB81 and its functional roles under abiotic stresses. Gene 2020, 753, 144803. [CrossRef] [PubMed]

32. Wang, F.; Ren, X.; Zhang, F.; Qi, M.; Zhao, H.; Chen, X.; Ye, Y.; Yang, J.; Li, S.; Zhang, Y.; et al. A R2R3-type MYB transcription factor gene from soybean, GmMYB12, is involved in flavonoids accumulation and abiotic stress tolerance in transgenic Arabidopsis. Plant Biotechnol. Rep. 2019, 13, 219-233. [CrossRef]

33. Zhao, L.; Li, M.; Xu, C.; Yang, X.; Li, D.; Zhao, X.; Wang, K.; Li, Y.; Zhang, X.; Liu, L.; et al. Natural variation in GmGBP1 promoter affects photoperiod control of flowering time and maturity in soybean. Plant J. 2018, 96, 147-162. [CrossRef] [PubMed]

34. Wang, F.; Chen, H.W.; Li, Q.T.; Wei, W.; Li, W.; Zhang, W.K.; Ma, B.; Bi, Y.D.; Lai, Y.C.; Liu, X.L.; et al. GmWRKY27 interacts with GmMYB174 to reduce expression of GmNAC29 for stress tolerance in soybean plants. Plant J. 2015, 83, 224-236. [CrossRef] 